\title{
Herlyn-Werner-Wunderlich syndrome - a rare genitourinary anomaly in females: a series of four cases
}

\author{
Mohd Ilyas ${ }^{1 A, B, C, D, E, F}$, Insha Khan $2 A, B, C, D, E, F$, Cimona L. Saldanha ${ }^{2 A, B, C, D, E, F}$ \\ 'Department of Radiodiagnosis and Imaging, Sher-I-Kashmir Institute of Medical Sciences, Srinagar, India \\ ${ }^{2}$ Department of Obstetrics and Gynaecology, Sher-I-Kashmir Institute of Medical Sciences, Srinagar, India
}

\begin{abstract}
We present case series of four patients with an important syndrome known as Herlyn-Werner-Wunderlich syndrome. Herlyn-Werner-Wunderlich syndrome is a rare congenital anomaly characterised by uterus didelphys with blind hemivagina and ipsilateral renal agenesis. It usually presents after menarche with progressive pelvic pain during menses secondary to haematocolpos. Awareness is necessary to diagnose and treat this disorder properly before complications occur. Magnetic resonance imaging is the preferred modality for the delineation of uterine malformation. When renal anomalies are encountered, a screening should also be made for congenital abnormalities of the reproductive tract and vice versa.
\end{abstract}

Key words: uterus didelphys, obstructed hemivagina, renal agenesis, haematometra, haematocolpos.

\section{Introduction}

Herlyn-Werner-Wunderlich (HWW) syndrome, recently known as obstructed hemivagina and ipsilateral renal anomaly (OHVIRA) syndrome, is a rare complex of structural abnormalities of the female urogenital tract. It is characterised by the triad of mesonephric duct-induced mullerian anomalies including: didelphys uterus, obstructed hemivagina, and ipsilateral renal agenesis. As per new classification, there are two types: type 1, a completely obstructed hemivagina, and type 2 , an incompletely obstructed hemivagina [1].

\section{Description of cases}

\section{Case 1}

An 18-year-old female patient presented with a history of secondary amenorrhea for four months with recurrent abdominal pain and lower abdomen swelling. Magnetic resonance imaging (MRI) was performed which revealed left haematometra, haematocolpos, and haematosalpinx with normal right uterine cavity with complementary sonography revealing absent left kidney (Figure 1).

\section{Case 2}

A 14-year-old young female, menarche at the age of 13 years, with gradually increasing pelvic pain. Ultrasound imaging of the abdomen revealed absent right kidney and uterus didelphys with right cavity dilated along with right fallopian tubes and normal left uterine cavity. The patient underwent MRI of the abdomen and pelvis, and findings thereof confirmed uterine didelphys and absent right kidney, and a diagnosis of HWW syndrome was made (Figure 2).

\section{Case 3}

A 26-year-old patient presented with history of irregular menses with recurrent lower abdominal pain and gradu-

Correspondence address:

Dr Mohd Ilyas, Department of Radiodiagnosis and Imaging, Sher-I-Kashmir Institute of Medical Sciences, Srinagar, India, 190011, e-mail: ilyasmir40@gmail.com Authors' contribution:

A Study design · B Data collection · C Statistical analysis · D Data interpretation · E Manuscript preparation · F Literature search · G Funds collection 

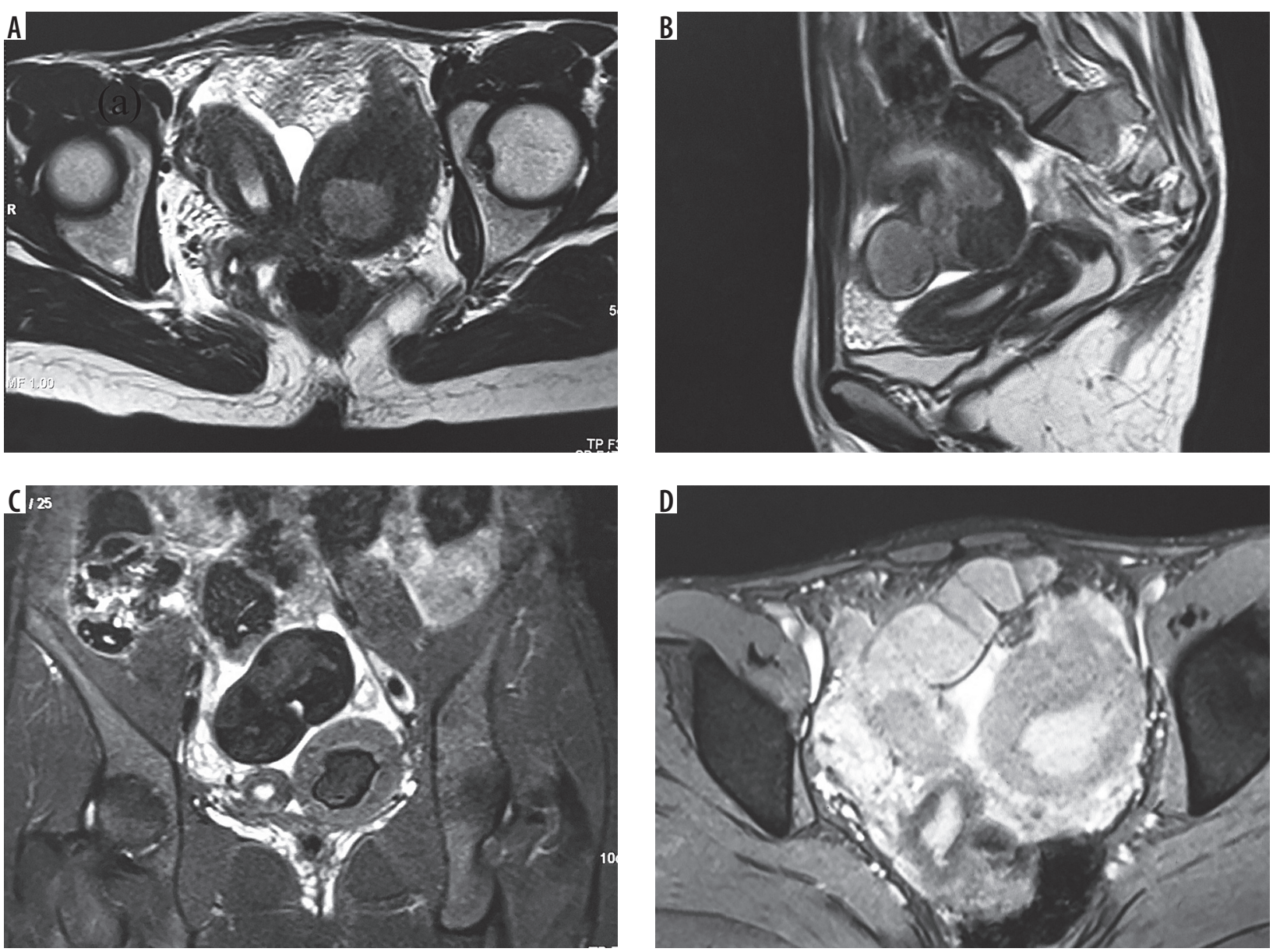

Figure 1. A) Axial magnetic resonance image (MRI) showing the normal right horn with dilated left uterine horn in a case of uterus didelphys. B) Sagittal image of the same patient showing the normal uterine left cavity. C) Coronal MRI showing the normal right uterine horn with haematometra-filled left uterine horn. D) Axial MRI of the same patient showing the dilated left uterine cavity along with dilated left fallopian tube with haematometra and haematosalpinx

al supra-pubic swelling for the past 2-3 months. She was married for two years and was unable to conceive. The patient was referred for MRI of the pelvis to our department, which revealed the findings of uterus didelphys with right haematocolpos and haematometra with absent right kidney (Figure 3).

\section{Case 4}

A 13-year-old patient presented with history of primary amenorrhoea, lower abdominal pain, and gradually increasing supra-pubic swelling. She was referred to our department for MRI scan of the pelvis, which revealed uterus didelphys with grossly dilated right hemivagina with blood products and normal left uterine cavity. Coronal scans revealed absent right kidney and normal left kidney (Figure 4).

\section{Discussion}

The urinary and genital systems arise from a common ridge of mesoderm ascending along the dorsal body wall, and they rely on normal development of the mesonephric system. Any anomaly of one system should prompt the evaluation of other system. HWW syndrome is rare syndrome arising due to maldevelopment of mesonephric and para-mesonephric ducts.

HWW syndrome typically presents after menarche with non-specific symptoms secondary to haematocolpos. The variation in presentation makes its diagnosis difficult and hence awareness regarding it is needed. MRI is suitable for diagnosis. HWWS is a combination of type III mullerian anomaly with mesonephric duct anomaly with vaginal septum. The classic renal manifestation of OHVIRA syndrome is ipsilateral renal agenesis, but reports of duplicated kidneys, dysplastic kidneys, rectovesical bands, or crossed fused ectopia have also been described [2]. Uterus didelphys with obstructed hemivagina is due to lateral non-fusion of the mullerian ducts with asymmetric obstruction, and it is almost always associated with renal agenesis ipsilateral to the side of obstruction. An important point to note in patients with HWWS is that renal agenesis is located ipsilateral to the dilated uterine cavity [3].

When suspecting a genitourinary anomaly, an initial screening ultrasound should be followed by MRI rather 

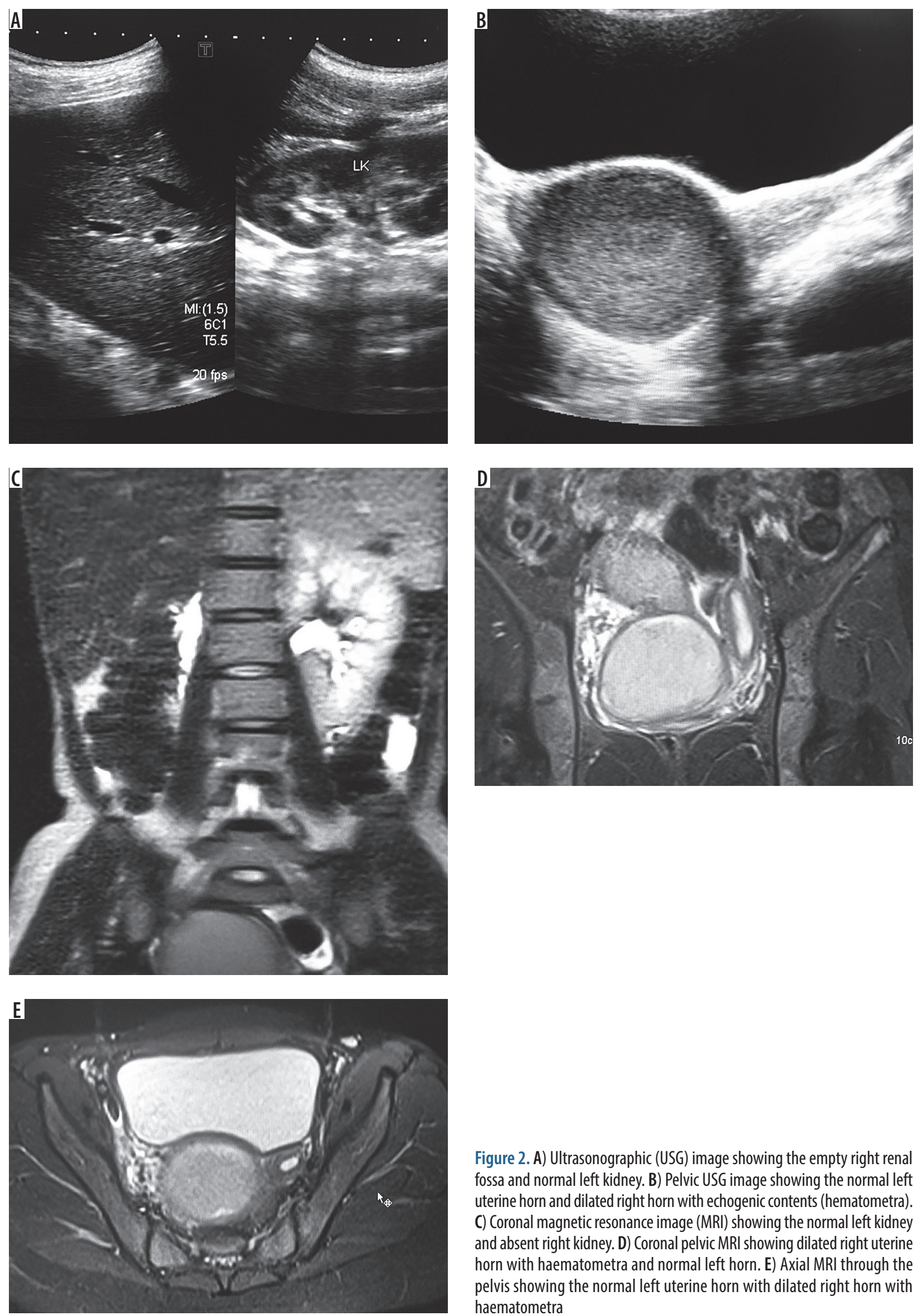

Figure 2. A) Ultrasonographic (USG) image showing the empty right renal fossa and normal left kidney. B) Pelvic USG image showing the normal left uterine horn and dilated right horn with echogenic contents (hematometra). C) Coronal magnetic resonance image (MRI) showing the normal left kidney and absent right kidney. D) Coronal pelvic MRI showing dilated right uterine horn with haematometra and normal left horn. E) Axial MRI through the pelvis showing the normal left uterine horn with dilated right horn with haematometra 

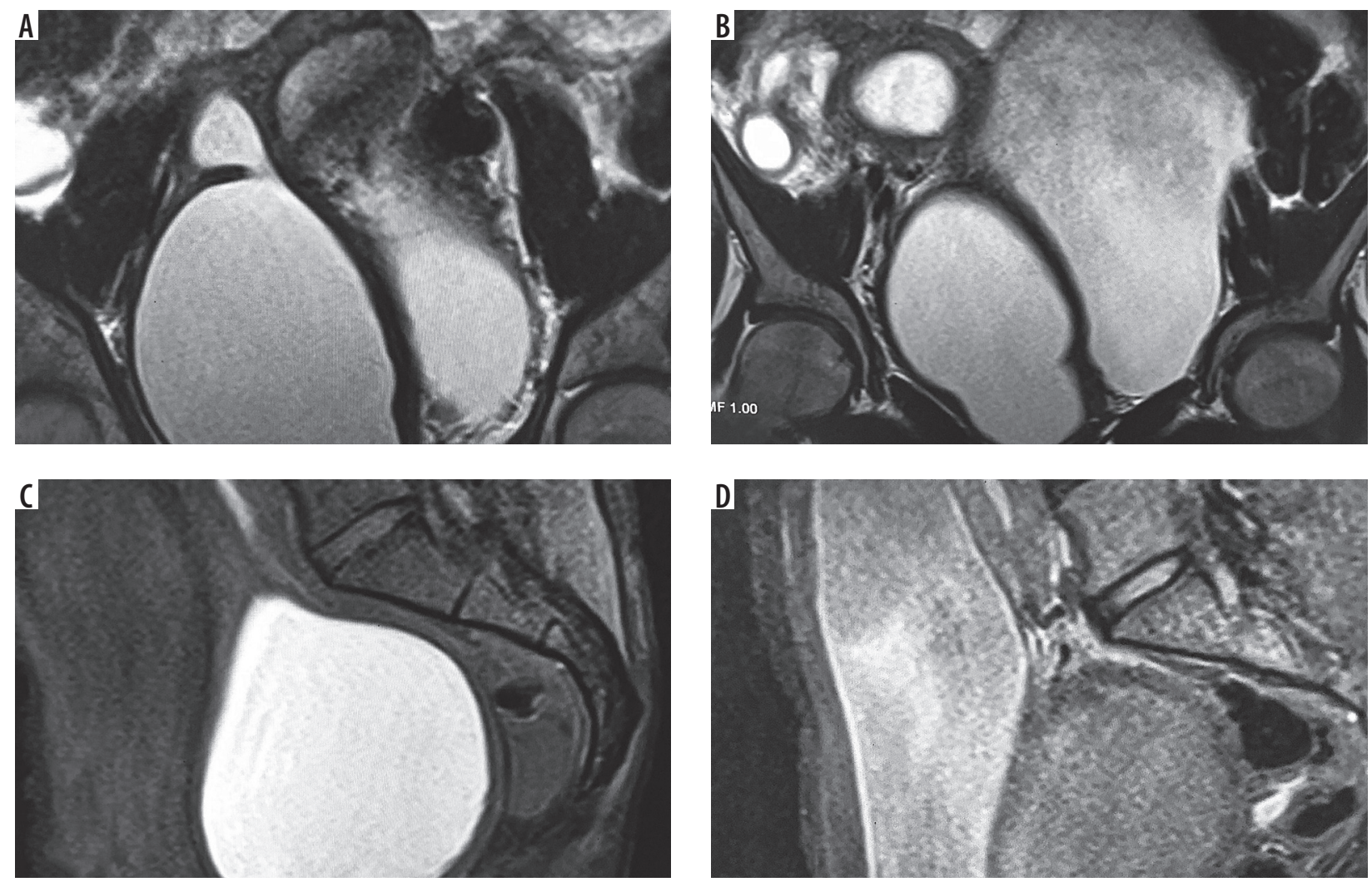

Figure 3. A) Coronal magnetic resonance image (MRI) showing the normal left uterine cavity and grossly dilated right uterine horn with internal haemorrhagic content. B) Coronal MRI in the same patient showing the dilated uterine horn and urinary bladder separately. C) Sagittal MRI showing the normal uterine cavity without haematometra. D) Sagittal MRI showing normal left uterine cavity and a part of dilated right uterine horn
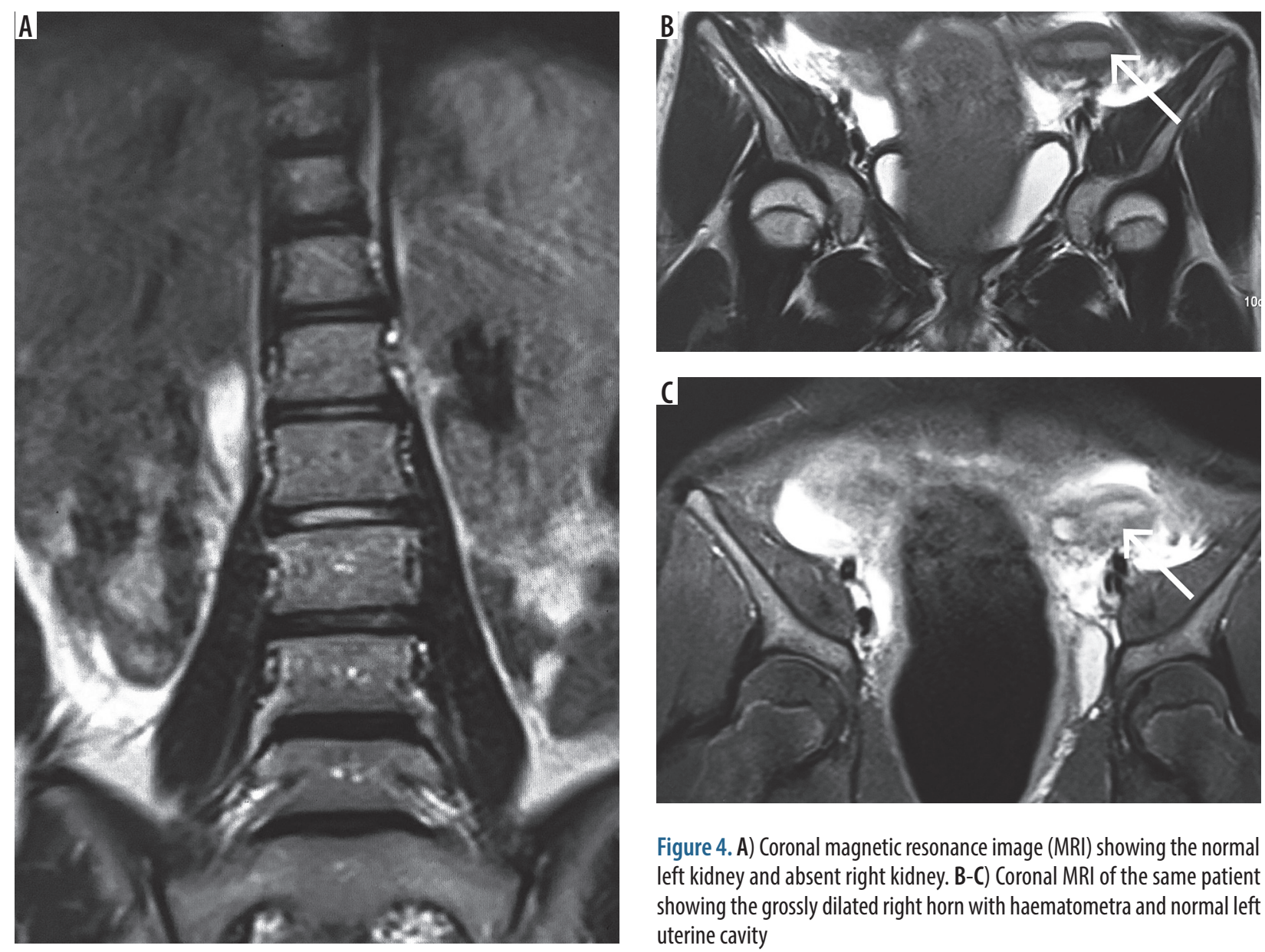

Figure 4. A) Coronal magnetic resonance image (MRI) showing the normal left kidney and absent right kidney. B-C) Coronal MRI of the same patient showing the grossly dilated right horn with haematometra and normal left uterine cavity 
than CT because the later involves radiation exposure, and many questions remain unanswered because of its limited soft-tissue resolution [4]. Considering the vast differential in uterovaginal anomalies, precise anatomical delineation of the uterus, tubes, cervix, and vagina is essential. The status of these reproductive organs and extent of endometriosis, pelvic inflammation, and adhesions can be well evaluated with MRI.

Laparoscopy remains the gold standard diagnostic modality with the additional advantages of therapeutic drainage of haematocolpos/haematometra, vaginal septectomy, and marsupialisation [5].

Laparoscopic vaginal septum excision is the treatment of choice for HWWS. A successful pregnancy is achieved eventually in $87 \%$ of patients, while $23 \%$ have the risk of abortion [6].

\section{Conclusions}

The infrequency of HWW syndrome complicates its diagnosis and hence clinicians should consider mullerian duct abnormalities among the differential diagnosis in young female patients presenting with abdominal symptoms. MR imaging of the pelvic organs and abdomen forms an important modality in the diagnosis of this anomaly to guide better treatment modality and avoid misdiagnosis of large haematometra/haematocolpos as endometriotic cysts.

\section{Conflict of interest}

The authors report no conflict of interest.

\section{References}

1. Zhu L, Chen N, Tong JL, et al. New Classification of Herlyn-WenerWunderlich Syndrome. Chin Me J (Engl) 2015; 128: 222-225.

2. Wu TH, Wu TT, Ng YY, et al. Herlyn-Werner-Wunderlich syndrome consisting of uterine didelphys, obstructed hemivagina and ipsilateral renal agenesis in a newborn. Pediatr Neonatol 2012; 53: 68-71.

3. Jeong JH, Kim YJ, Chang CH, Choi HI. A case of Herlyn-WernerWunderlich syndrome with recurrent hematopyometra. J Womens Med 2009; 2: 77-79.

4. Asha B, Manila K. An unusual presentation of uterus didelphys with obstructed hemivagina with ipsilateral renal agenesis. Fertil Steril 2008; 849: e9-10.
5. Angotti R, Molinaro F, Bullota AL, et al. Herlyn-Werner-Wunderlich syndrome: An "early" onset case report and review of literature. Int J Surg Case Report 2015; 11: 59-63.

6. Park NH, Park HJ, Park CS, Park SI. Herlyn-Werner-Wunderlich syndrome with unilateral hemivaginal obstruction, ipsilateral renal agenesis and contralateral renal thin GBM disease: A case report with radiological follow up. J Korean Soc Radiol 2010; 62: 383-388. 